\title{
MRS JOURNAL HIGHLIGHTS
}

MRSS ENERGY Q

SUSTAINABILITY
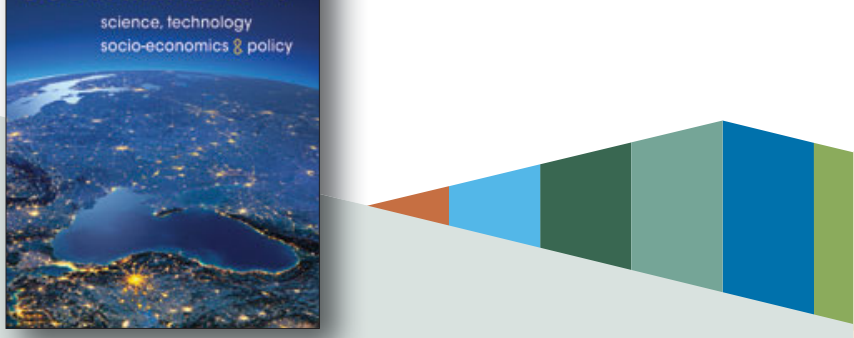

Carbonyl-coordinating polymers for high-voltage solidstate lithium batteries: Solid polymer electrolytes

Hongli Xu, Jingbing Xie, Zhongbo Liu, Jun Wang, Yonghong Deng

Solid polymer electrolytes are a crucial class of compounds in the next-generation solid-state lithium batteries with high safety and extraordinary energy density. This review highlights the importance of carbonyl-coordinating polymer-based solid polymer electrolytes in next-generation safe and high-energy density lithium-metal batteries, unraveling their synthesis, sustainability, and electrochemical performance. doi.org/10.1557/mre.2020.3

\section{Deep decarbonization efforts in Norway for energy sustainability}

\section{Truls Norby, Emil H. Jensen, Sabrina Sartori}

Norway is energy self-sufficient with ample renewable energy resources while exporting large amounts of fossil fuels. The country is in a unique position to fully decarbonize its energy use and significantly contribute to deep decarbonization globally, but Norway's small population and economy limit that impact. The authors discuss efforts toward its own and the Paris and Katowice meetings' ambitious goals of a carbon-neutral society. doi.org/10.1557/mre.2019.12

Applying insights from the pharma innovation model to battery commercialization - pros, cons, and piffalls

\section{Eve D. Hanson, Samir Mayekar, Vinayak P. Dravid}

A carbon-neutral and sustainable society necessitates the widespread use of battery technologies that are efficient, effective, and economical. Lower-cost and more energy-dense battery technology can help solve many energy challenges. However, bringing advanced energy materials to market in the United States remains a formidable challenge. The authors show how the pharmaceutical industry overcame similar technical challenges and offer comparisons for successful commercialization in the US battery industry.

doi.org/10.1557/mre.2017.12 mrs.org/publications-news

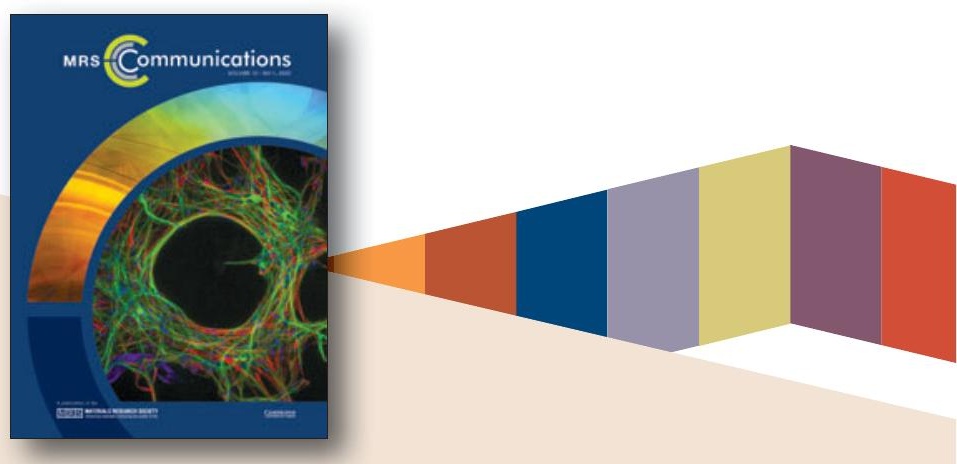

The structure and mechanical properties of $\mathrm{Cu}_{50} \mathrm{Ni}_{50}$ alloy nanofoams formed via polymeric templating

\section{Chang-Eun Kim, Raheleh M. Rahimi, David F. Bahr}

Interest in nanofoams based on template precursors enable the synthesis of transition-metal structures with controlled cell size and grain. Using electrochemical routes, it is possible to control the porosity based on the design of the polymer template while controlling the alloy composition. doi.org/10.1557/mrc.2020.16

\section{$\mathrm{N}$-dimensional optics with natural materials}

\section{Giulia Guidetti, Fiorenzo G. Omenetto}

The nature-inspired design of materials enables the discovery of new phenomena and the harnessing of smart analytical methods. Typical biomaterials and biomolecules can provide new insight toward the utilization in optical systems as organized assemblies, providing a medium for the electromagnetic phenomena.

doi.org/10.1557/mrc.2020.23

\section{Phenolic antioxidant-incorporated durable perovskite} layers and their application for a solar cell

\section{Koki Suwa, Takeo Suga, Kenichi Oyaizu, Hiroshi Segawa, Hiroyuki Nishide}

Hybrid materials have been gaining ground because of the synergy and interplay of bandgap and mobility phenomena across molecular species. Key is the knowledge of the interfacial and transport properties of the radical/electron mobile species and their spatiotemporal properties during device operation. The authors lead the way by incorporating the use of phenolic species.

doi.org/10.1557/mrc.2020.25

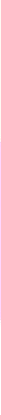

